\title{
Chronic right lower abdominal quadrant pain and the vermiform appendix
}

\author{
Vengayil Sukumaran ${ }^{1}$, Bhavuray Teli ${ }^{1}$, Sreekant Avula $^{1}{ }^{*}$, Jagadeesh Pavuluru $^{1}$, Satish Arakeri $^{2}$
}

\author{
${ }^{1}$ Department of Surgery, DMWIMS, Wayanad, Kerala, India -673577 \\ ${ }^{2}$ Department of Pathology, DMWIMS, Wayanad, Kerala, India -673577
}

Received: 5 November 2015

Revised: 7 January 2016

Accepted: 9 January 2016

\author{
*Correspondence: \\ Dr. Sreekant Avula, \\ E-mail: drsreekanth.ms@gmail.com
}

Copyright: (C) the author(s), publisher and licensee Medip Academy. This is an open-access article distributed under the terms of the Creative Commons Attribution Non-Commercial License, which permits unrestricted non-commercial use, distribution, and reproduction in any medium, provided the original work is properly cited.

\begin{abstract}
Background: Although chronic right lower quadrant (RLQ) abdominal pain is a common clinical condition, the diagnosis and treatment is still under debate. In spite of literature evidence, existence of recurrent or chronic appendicitis continues to be debated-especially, whether chronic appendicitis really exists and whether it can explain the persistent or chronic right lower quadrant abdominal pain. We study here whether undiagnosed chronic RLQ abdominal pain can be due to appendix and whether it will be relieved after elective laparoscopic appendicectomy. In literature there are quite a few studies on this, but all are limited by smaller sample size.

Methods: 200 patients, 12-60 years of age, with undiagnosed chronic RLQ abdominal pain underwent diagnostic laparoscopy and appendicectomy. Twenty patients were excluded from the final analysis because of the presence of an obvious non-appendicular pathology also. Patients were followed up for 6 months at regular intervals (one, three and six month) and were assessed for pain relief. The association between clinical outcome and histopathological outcome was studied.

Results: The histopathological examination of appendices revealed appendicitis in all patients. Intra-operative evidences of chronic appendicitis were seen in $160(88.8 \%)$ patients. $175(97.2 \%)$ patients were completely pain free on follow up and only $5(2.8 \%)$ patients had persistence of pain.

Conclusions: Patients with chronic RLQ abdominal pain (without obvious diagnosis on preoperative evaluation) can safely undergo exploratory laparoscopy and appendicectomy. Chronic appendicitis is a diagnosis of exclusion with intra-operative and histopathological evidence. Patients with RLQ abdominal pain and right iliac fossa (RIF) tenderness with otherwise normal findings on sonology are having chronic or recurrent inflammation of the vermiform appendix and are fully curable by laparoscopic appendicectomy.
\end{abstract}

Keywords: Chronic, Laparoscopic, Appendicectomy, Appendicitis, Abdominal pain

\section{INTRODUCTION}

Recently, chronic right lower quadrant (RLQ) abdominal pain has drawn more attention because of its high incidence, significant morbidity and significant costs to health care. Patients often complain of pain in the RLQ of abdomen, of months to year's duration for which various diagnostic and therapeutic interventions were carried out with no relief. The vermiform appendix is one of the commonest sites of inflammatory pathology in the abdomen. ${ }^{1}$ Traditionally the only well documented and clinically significant one among the inflammations of the appendix has been the "acute appendicitis". But, the lack of objectivity in the diagnostic algorithms in case of 
appendicitis in general, has led to many clinical confusions and increased patient morbidity. On one hand, there is a huge number of cases which are underdiagnosed and on the other, a significant series of cases with a high negative appendicectomy rate (NAR). In this context, we believe, that the basic fact about the inflammatory disorders of the appendix is that these can present with a spectrum of character and severity, ${ }^{3,4}$ so that, the accurate positive diagnosis of appendicitis cannot be defined just by traditional parameters like tachycardia, rebound tenderness, leukocytosis, the Alvardo scoring system, ultrasonogram (USG) or even by computerized tomography (CT) of the abdomen. Here, we present a large series of pathologically proven chronic or recurrent inflammation of the vermiform appendix, all of which presented with chronic RLQ abdominal pain, without the traditionally relied upon clinical features and sonological evidence to support, but with an excellent cure rate fallowing laparoscopic appendicectomy.

\section{METHODS}

It was a prospective interventional study, carried out from January 2013 to June 2015. Patients with RLQ abdominal pain ranging from 6 weeks to 5 years in duration, which remained undiagnosed, were chosen for the study.

\section{Inclusion criteria}

Patients aged 12-60 years, suffering from chronic or recurrent RLQ abdominal pain for more than 6 weeks who had been treated conservatively by antibiotics and analgesics without the classical clinical and/or sonological features of acute appendicitis.

\section{Exclusion criteria}

Patients with chronic RLQ abdominal pain but also with a history of chronic back pain (previous spine surgery, diagnosed disc prolapse), previous abdominal surgery (except diagnostic laparoscopy or laparoscopic sterilization), specific gastrointestinal disorders (Crohn's disease, ulcerative colitis, Koch's abdomen), gynecological diseases (pelvic inflammatory disease, endometriosis) and known urological diseases (renal stones, ureteric stones). 20 patients were excluded postoperatively as they had associated uterine and/or adnexal lesions as well.

All patients were preoperatively subjected to haemogram, renal function test, random blood sugar, urine routine examination, abdominal USG and chest radiography. All 200 patients underwent thorough diagnostic laparoscopy with appendicectomy and those with obvious nonappendicular pathology also, which could interfere with final analysis, were excluded.

All specimens were subjected to HPE. All patients were followed up in the outpatient department for up to 6 months (one, two and six month). Patients were scored for pain as completely relieved and pain persistent/increased. The clinical outcome was studied in relation to pathological reports.

\section{RESULTS}

Total 200 patients were included in the study. 20 patients were excluded after diagnostic laparoscopy due to coexisting PID/ovarian cyst. Of the 180 patients who were finally included, $72(40 \%)$ were male and 108 $(60 \%)$ were female (Figure 1) with a median age of 25.5 (Range: 12-60) years. 55\% (99) of patients fall into the age group of 21-30 years. All patients had various degrees of right iliac fossa (RIF) tenderness. None had tachycardia or leukocytosis. USG abdomen detected probe tenderness in $25 \%$ patients and in these, ten patients had sonologically visible appendix of $6-10 \mathrm{~mm}$ diameter. $88.8 \%$ patients showed features of chronic appendicitis such as pale, narrow, fibrosed appendix with periappendiceal adhesions to various extents. At the end of 6 months, 175 out of $180(97.2 \%)$ patients were completely pain free. There were $5(2.8 \%)$ patients with pain remaining even after 6 months (Figure 2). There was no patient whose pain was worse than before. NAR in this series is zero. 144 patients had histopathological proof of chronic inflammation of the appendix. 36 patients showed pathological changes suggestive of acute on chronic appendicitis (Figure 3).

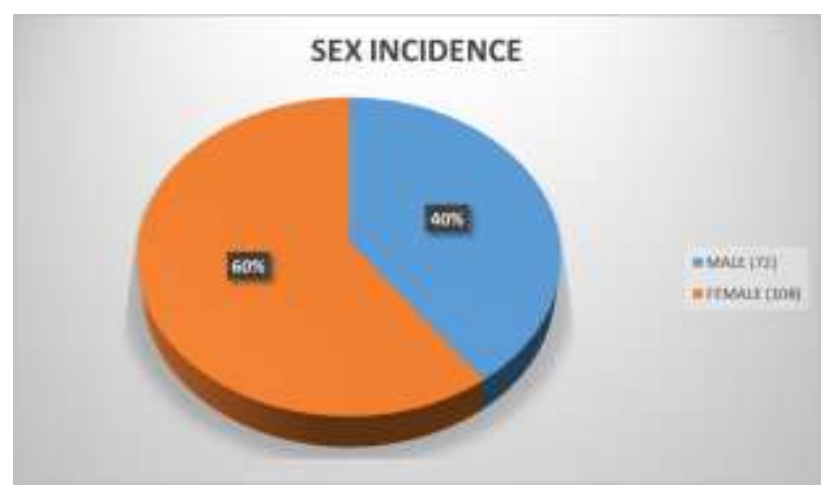

Figure 1: Sex incidence.

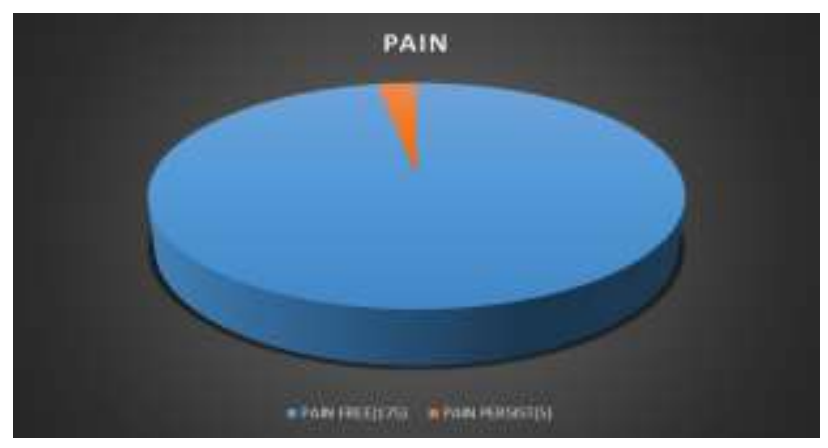

Figure 2: No. of patients with pain free and pain persisting. 


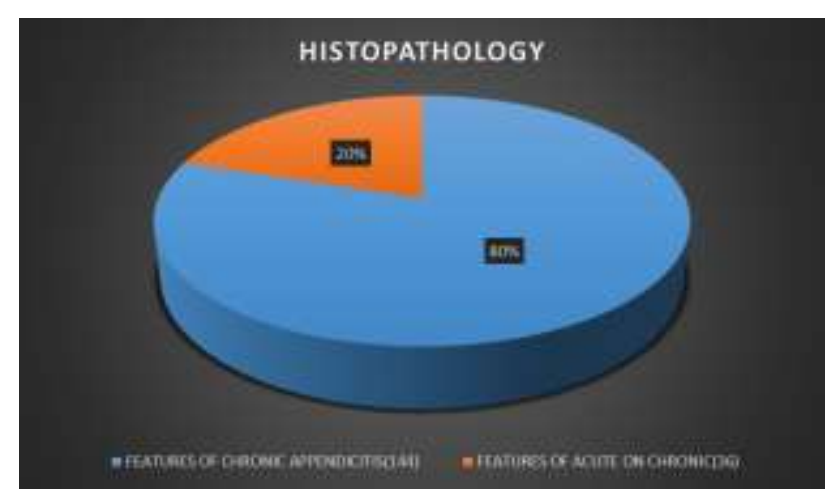

Figure 3: Histopathological features of appendicitis.

\section{Acute on chronic appendicitis}

\section{Gross findings}

The wall thickness is increased/ decreased; presence of congested blood vessels on surface, color is dull sheen.

\section{Microscopy}

Section studies show mucosa with mixed inflammatory infiltrate composed of both neutrophils and lymphocytes with equal proportion. Fibrosis may be present.

\section{Chronic appendicitis}

\section{Gross findings}

The wall thickness is decreased, presence of congested blood vessels on surface, pale looking appendix with shiny sheen appearance.

\section{Microscopy}

Section studies show normal mucosa with predominant lymphocytic infiltration with a few scattered neutrophils and eosinophils. Fibrosis is present. Fatty infiltration in muscular layer and hypertrophic neural bundles seen predominantly.

\section{DISCUSSION}

Consensus regarding a chronic inflammatory process in the vermiform appendix as a cause of chronic RLQ abdominal pain is lacking. Hitherto, the only well recognized form of inflammation of the appendix is the acute appendicitis. ${ }^{2}$ Even though it is widely accepted that the diagnosis of acute appendicitis is largely clinical, the low confidence level for this particular diagnosis for any given clinician has led to unnecessary dependence on investigative modalities like USG or CT scan, a state which has led to a huge percentage of under-diagnoses and under-treatment, there by constituting an unacceptable level of morbidity in patients suffering from RIF pain. The recurrence rate in sub optimally treated (by intervention other than surgical) appendicitis is of great concern too.

There is divergence of opinion regarding the clinical entity of chronic appendicitis, as a cause of recurrent pain in the RLQ. These patients may not present with the traditionally accepted clinical features of inflamed appendix. Instead, they complain of months to years of RLQ abdominal pain. They may have had multiple medical evaluations and conservative treatment in the past for this pain. The patients may describe an initial episode with more classic symptoms of acute appendicitis, for which no treatment was received or treated conservatively. The diagnosis of chronic appendicitis can be difficult, as hematology and radiological studies are typically normal. HPE evaluation confirms the diagnosis of chronic inflammation. ${ }^{3-4}$ As the diagnosis is often uncertain preoperatively, laparoscopy can be a useful tool to allow exploration of the abdomen. ${ }^{5}$

Recurrent appendicitis refers to a pattern of symptoms with mild, self-limited attacks of RLQ pain that typically last for hours before resolving spontaneously with histological evidence of appendicitis.

Chronic appendicitis refers to constant well-localized RLQ abdominal pain and tenderness with no other identifiable pelvic or abdominal disease. If appendicectomy completely relieves the pain and pathologic findings include chronic inflammation of the appendix, then the diagnosis of chronic appendicitis is confirmed. ${ }^{6,7}$

In our study, pain abdomen was the universal complaint in all 180 patients $(100 \%)$. Anorexia, nausea/vomiting were present in $10 \%$ patients. The recurrence rate in sub optimally treated (by interventions other than surgical) acute appendicitis (due to failure to confirm the diagnosis) and the suffering due to undiagnosed chronic RLQ abdominal pain are unacceptably high. Overemphasis on clinical parameters like tachycardia, rebound tenderness and laboratory parameters such as leukocytosis and sonological evidence has eventually led to a doubtful diagnosis and a harmful increase in resorting to CT scan of abdomen, at the end of all, the patient remains undiagnosed and untreated for an elusive appendicitis. While relying too much on the traditionally overemphasized clinical and investigative parameters to make a diagnosis of an inflammatory process in the appendix, we tend to forget that any inflammation can present with varying degrees of severity and character, so that the clinical and laboratory interpretation must not be restricted by too rigid and dogmatic concepts.

In our series of 200 patients who presented with chronic RLQ abdominal pain, the only clinical sign of a probable inflammatory process in the RIF was a varying degree of RIF tenderness. None of them had tachycardia (as defined by a resting heart rate of more than 100 beats/min), rebound tenderness or elevated body 
temperature. No patients showed leukocytosis (as defined by a total leucocyte count of more than 11000 cells $/ \mathrm{mm}^{3}$ ). But $35 \%$ of patients had a relative neutrophilia (defined as a neutrophilic predominance of the grade $75 \%$ or more). $5 \%$ of patients with chronic RLQ abdominal pain had a sonologically demonstrated increase in the size of the appendix with features of inflammation. All 200 patients underwent exploratory laparoscopy. 20 patients had a demonstrable non-appendicular pathology also in and around the pelvis. All others had an otherwise normal abdomen as for as laparoscopy could demonstrate, except for the fact that $88.8 \%$ of patients had features of chronic inflammatory process in and around the appendix as described by a narrowed, pale and fibrosed appendix with periappendiceal adhesions of varying degrees. Histopathological analysis of the appendicular specimen proved that, all appendices were pathological and so the NAR is zero. $97 \%$ of patients had complete cure of RLQ abdominal pain. $3 \%$ had persistent pain presumably due to stump appendicitis.

Laparoscopy is changing the approach towards chronic RLQ abdominal pain. It is a diagnostic as well as a therapeutic tool. An exploratory and interventional (appendicectomy) study was conducted by Kolts RL et al in 44 pediatric patients having recurrent RLQ abdominal pain. ${ }^{8} 15$ patients $(34 \%)$ had abnormalities other than appendix during exploratory laparoscopy. Out of 44 patients, $32(72.7 \%)$ were found to have histologic abnormalities in the appendix on 2 years follow-up, complete resolution of abdominal pain occurred in 25 $(56.8 \%)$ patients, partial resolution in 6 patients $(13.6 \%)$ and no response in 13 patients $(29.6 \%)$. Hence, the conclusion of the study was that laparoscopic appendicectomy performed during exploratory laparoscopy is a useful treatment for chronic or recurrent RLQ abdominal pain and can also be a good diagnostic tool for other diseases processes in the abdomen.

Another study was done by Roumen RMH et al for chronic RLQ abdominal pain. ${ }^{9}$ It was a double-blinded randomized control trial. Of 40 patients, 18 patients underwent laparoscopic appendicectomy and 22 patients only diagnostic laparoscopy. Post-operative pain scores and histopathology of appendix were compared between the two groups. Out of 18 patients in the laparoscopic appendicectomy group, 7 appendices were found to be normal and 11 showed signs of appendicopathy. At six month follow up they observed that higher proportion of patients in the appendicectomy group had significant improvement in pain than the other. It was concluded that persistent or recurrent RLQ pain can be treated successfully by elective laparoscopic appendicectomy in properly selected cases and there was no significant relationship between post-operative pain scores and the histopathology findings of the appendices.

Al-Araji KS et al performed appendicectomy in 58 patients with chronic or recurrent RLQ pain of 3 months to 3 years duration. ${ }^{10}$ Out of 58 patients, $54(93.1 \%)$ had gross changes of chronic inflammation in the appendix and surrounding tissues. Only 17 specimens were subjected for HPE and 16 showed chronic inflammatory changes. On follow-up, $56(96.5 \%)$ patients were completely pain-free.

In a study by Charles $\mathrm{C}$ et al ten patients underwent an elective appendicectomy for chronic RLQ pain. ${ }^{11}$ No macroscopic abnormalities were seen during surgery. Histopathological analysis showed inflammatory changes in eight out of ten patients. The conclusion of the study was that significant reduction of pain can be achieved after an appendicectomy in all patients suffering from chronic RLQ abdominal pain.

In a recent study on chronic RLQ abdominal pain by Gedam MC et al. they performed laparoscopic appendicectomy in 30 patients. $^{12}$ In this study, 22 $(73.4 \%)$ out of 30 patients had recurrent appendicitis and $26(86.6 \%)$ patients had complete pain relief after appendicectomy at 6 months follow up. The conclusion of the study was that laparoscopy is an invaluable tool in diagnosing conditions other than chronic appendicitis existing concurrently in patients with chronic RLQ pain. There is no relation between the relief of pain and histopathology of the appendix. Nevertheless chronic RLQ abdominal pain can be relieved by performing laparoscopic appendicectomy.

Another study conducted by Popovic et al 41 out 53 cases of chronic RLQ abdominal pain underwent laparoscopic appendicectomy. ${ }^{13}$ The study concluded that long term results are similar in both laparoscopic appendicectomy and without appendicectomy groups. There was no relation between clinical improvement and histopathology of appendices. The study also mentioned that appendicectomy should be performed even though there is no macroscopically visible reason because of the intraluminal changes.

In another study by Charlesworth $\mathrm{P}$ et al in pediatric population, 16 children underwent laparoscopic appendicectomy for chronic RLQ abdominal pain. ${ }^{14}$ There was early improvement in $14 / 16$ patients. This figure had improved to a $100 \%$ on extended fallow up. Median follow-up was 19 months (range 1-47). The conclusion of the study was that symptomatic improvement can be expected to be $88 \%$ immediately and up to $100 \%$ in the long term. The study supports laparoscopic appendicectomy in all patients presenting with chronic RLQ pain in spite of negative hematological and radiological investigations.

\section{CONCLUSION}

The vermiform appendix is a common site of inflammatory disorder and is a major surgical cause of morbidity. Other than the acute appendicitis, appendicular inflammation can also present with chronic or recurrent RLQ abdominal pain of milder degrees but 
without tachycardia, leukocytosis and sonological evidence. The hematological and radiological studies do not contribute the diagnosis of chronic or recurrent appendicitis. But intra-op findings on laparoscopy and histopathology of the removed appendix confirm the diagnosis. The diagnosis of this entity is largely clinical and laparoscopic appendicectomy is curative.

Funding: No funding sources

Conflict of interest: None declared

Ethical approval: The study was approved by the institutional ethics committee

\section{REFERENCES}

1. Makaju R, Mohammad A, Shakya A. Acute appendicitis: analysis of 518 histopathologically diagnosed cases at the Kathmandu University Hospital, Nepal. KUMJ. 2010;8:22730.

2. Chen L, Crawford JM. The gastrointestinal tract in robbins pathological basis of disease $7^{\text {th }} \mathrm{Ed}$. Philadelphia: Saunders an imprint of Elsevier Inc. 2005:870-2.

3. Jones AE, Phillips AW, Jarvis JR, Sargen K. The value of routine histopathological examination of appendicectomy specimens. BMC Surg. 2007;10:7.

4. Ramraje S, Bharambe B, Ansari S, Kamble T, Goel A. Histological audit of appendicectomy specimens. Intern J Basic Appl Med Sci. 2012;2:146-9.

5. Smink DS, Soybol DI. Appendix and appendectomy. Maingot's abdominal operations. $11^{\text {th }}$ ed. McGraw-Hill Companies;2007:606.

6. Ziad T, Awad W, Eubanks S. Laparoscopic Appendectomy. Mastery of surgery volume 1. $5^{\text {th }}$ ed.;2007:1435.
7. Sarosi GA, Turnage RH. Appendicitis. Feldman: Sleisenger \& Fordtran's gastrointestinal and liver disease. $8^{\text {th }}$ ed. Saunders;2006:2608.

8. Kolts RL, Nelson RS, Park R. Exploratory laparoscopy for recurrent right lower quadrant pain in a pediatric population. Pediatr Surg Int. 2006;22:247-9.

9. Roumen RM, Groenendijk RP, Sloots CE. Randomized clinical trial evaluating elective laparoscopic appendicectomy for chronic right lower quadrant pain. Br J Surg. 2008;95:169-75.

10. Al-Araji KS. Recurrent "Chronic" appendicitis. Is it a myth? Is appendicectomy indicated? Med J Babylon. 2006;3:51-5.

11. Charles C, Rossem V, Treskes K, Loeza DL, Anna AW, Geloven V. Laparoscopic appendicectomy for chronic right lower quadrant pain. Int $\mathrm{J}$ Colorectal Dis. 2014;29:1199-202.

12. Gedam MC, Kulkarni AA, Lanjewar SM, Ingle L. Role of elective laparoscopic appendicectomy in chronic right lower quadrant pain: a prospective \& interventional study. Int Surg J. 2015;2:200-3.

13. Popovic D, Kovjanic J, Milostic D, Kolar D, Stojakovic D, Obradovic Z et al. Long term benefits of laparoscopic appendectomy for chronic abdominal pain in fertile women. Croat Med J. 2004;45(2):171-5.

14. Charlesworth P, Mahomed A. Diagnostic laparoscopy and appendicectomy for children with chronic right iliac fossa pain: an aggregate analysis. J Pediatr Surg Special. 2010;3:9-14.

Cite this article as: Sukumaran V, Teli B, Avula S, Pavuluru J, Arakeri S. Chronic right lower abdominal quadrant pain and the vermiform appendix. Int Surg J 2016;3:221-5. 